\title{
The output code of a visual fixation
}

\author{
LIDEWIJ L. VAN DUREN and ANDRIES F. SANDERS \\ Vrije Universiteit, Amsterdam, The Netherlands
}

\begin{abstract}
The present experiment investigated whether a saccadic eye shift is delayed until perceptual and postperceptual processing of the fixated signal are completed. Two signals were presented with an angular distance of $100^{\circ}$. Subjects inspected the left signal (SL) first and then made an eye-and-head movement to the right signal. Various task conditions were manipulated in such a way, that, although SL remained the same, the information it contained either allowed for perceptual processing only or also included postperceptual processing (classification, response selection). In addition, the signal quality of SL was manipulated in all conditions. The fixation duration of SL was affected by signal quality and not by task condition. It was concluded that a saccade is initiated upon completion of perceptual processing of a signal.
\end{abstract}

Processing during a visual fixation of a signal may vary from mere registration of sensory information to completion of all required central processing of that signal. The present study investigated the extent to which central processing demands determine the duration that a signal is fixated in advance of a saccadic shift to a subsequent signal. In other words, the question is whether fixation duration depends on the total amount of processing a signal requires or on the nature of the processing demands (i.e., some types of demands might affect the duration, and others might not), or whether there is a constant fixation duration, irrespective of the processing demands.

According to one extreme possibility, a saccade is initiated immediately upon sensory registration of information, for the simple reason that subsequent central processing of the signal does not require the presence of the physical input. Central processing may continue during the saccade and, eventually, during the next fixation (Russo, 1978). In the latter case, the signal presented at that next fixation has to be processed in addition to what still remains from the first signal; consequently, processing requirements of subsequent inputs accumulate. In order to avoid accumulation, the initiation of the saccade might therefore be delayed until central processing of the fixated signal is at least partly completed. The consequence would be that processing requirements of a signal have at least some effect on its fixation duration. Yet, the effect would merely reflect a top-down strategy to prevent subsequent overload, which implies that the effects should disappear if overload were prevented otherwise.

According to another extreme possibility a saccade is triggered only by the completion of a more elaborate internal code. This view suggests that all processes required to arrive at that code must always be completed before

The authors are grateful to D. E. Irwin for a critical reading of the paper. Correspondence should be addressed to L. van Duren, Faculteit der Psychologie en Pedagogische Wetenschappen, De Boelelaan 1111, 1081 HV Amsterdam, The Netherlands. a saccade can start. This bottom-up view is in line with notions of processing in discrete stages, whereas the topdown view corresponds with continuous flow models (Miller, 1988; Sanders, 1990).

From research on reading, it has become clear that cognitive processing is actually reflected in fixation duration. Both word frequency (Inhoff, 1984) and predictability of a word in a sentence (Ehrlich \& Rayner, 1981; Zola, 1984) have an effect on fixation duration in reading. However, a more detailed model for the sequence of events during a fixation in reading is not available (Rayner \& Pollatsek, 1987). It is clear that the finding that there are at least some effects of central processing demands on fixation duration can be explained by either of the discussed theoretical alternatives.

Researchers using tasks less complex than reading also found that at least some cognitive processing is completed before the saccade is initiated. Irwin carried out several experiments in which two signals were presented at two successive fixations. These signals were either two dot patterns that had to be compared (Irwin, 1991) or an array of letters during the first fixation and a partial report cue during the second fixation (Irwin, 1992). The results suggest that the contents of successive fixations are not compared on the basis of raw sensory information but on an encoded, more abstract representation of the signals. In turn, this suggests that there is indeed more elaborate encoding during a fixation. In a series of experiments, Sanders and Houtmans (1985) studied the extent to which perceptual processing is completed prior to the start of a saccade. They used a paradigm in which two signals were presented with an angular distance of $45^{\circ}$. Subjects first fixated the left signal (SL), which consisted of either an intact or a degraded digit. Following the fixation of SL, an eye movement was made to the right signal (SR), which was always an intact digit. A trial ended with a same/different response with respect to SL and SR. The results showed that the effect of the signal quality of SL on the duration of its fixation (TL) was about as large as the effect of the signal quality on reaction time (RT) in 
a control condition in which traditional choice reactions were required. This was taken as evidence that a saccade can start only upon completion of stimulus encoding. Subsequent experiments showed that it was difficult to force subjects by instruction to leave SL at an earlier stage of processing. When asked to start the saccade in response to a tone that was presented briefly in advance of SL, some subjects were not able to obey that instruction at all (they simply completed encoding of SL before starting the saccade), whereas the remaining subjects obeyed only at a certain proportion of trials. The problems that the subjects had in postponing perceptual processing of SL were considered as strong evidence that encoding a signal is tied to its fixation.

Still, the conclusion that an encoded stimulus is the invariable output code of a fixation is not justified. In the studies by Sanders and Houtmans (1985), as well as in subsequent research on this issue (Hansen \& Sanders, 1988), two separate signals were compared across successive fixations followed by a same/different response. On one hand, a same-different task is unsuitable to study effects of postperceptual processing on fixation duration, simply because the processing requirements of SL are limited to perceptual analysis in the same-different task. Postperceptual processing (e.g., classification, response selection) may be tied to the fixation of a signal as well, but this cannot be concluded from a same-different task. On the other hand, the completion of perceptual processing during the fixation in a same-different task may be due to the specific requirements of that task. For example, it might be advantageous to prepare the most suitable code of SL for subsequent comparison with SR prior to the start of the saccade, since postponing encoding of SL might disturb the subsequent comparison. This last possibility is obviously related to a strategic top-down view, which suggests that when a task does not require a comparison between SL and SR, stimulus encoding of the first signal might not be necessarily needed in advance of the start of the saccade.

The present experiment aimed at determining, therefore, whether the results of previous studies (Hansen \& Sanders, 1988; Sanders \& Houtmans, 1985) have some generality beyond a same-different task. This was accomplished by comparing the fixation duration of SL in a same-different task with that in three other tasks. In all four tasks, SL always consisted of the same stimulus (i.e., either one of two digits), whereas the requirements at the fixation of SR varied. In one alternative, SR also consisted of a digit: The subjects determined the sum of SL and SR and decided whether the result was odd or even. A difference between this summation task and the same-different task was that, in principle, establishing a physical code of SL sufficed in the same-difference task, whereas the summation task required a name code (Posner, 1978). The question is, then, whether or not this difference is reflected in the fixation duration. In two other tasks, postperceptual processing could occur in principle while fixating SL. In one task, SL had to be classified as larger or smaller while SR showed how the larger and the smaller signal were mapped onto either one of two response keys at that particular trial. Thus, in principle, classification of SL might be completed during TL. In the final task response selection could be completed during TL, since SR consisted of a go or a no-go signal indicating whether or not a choice reaction to SL should be carried out.

If all central processing of a signal is tied to its fixation, the last two tasks should show a longer TL than that for the summation task due to additional processing demands (target classification, response choice). In turn, the summation task should show a longer TL than that for the same-different task, reflecting the difference between establishing a physical code and a name code. Alternatively, TL might be longest in the same-different task if stimulus encoding is completed only in case of a subsequent comparison between SL and SR, as in the samedifferent task. Still another alternative is that perceptual processing is tied to a fixation, whereas postperceptual processing is carried out during a saccade or a next fixation. In that case, the differences between the tasks should not affect TL at all.

\section{METHOD}

\begin{abstract}
Apparatus
The experiment took place in a sound-attenuated dimly lit cubicle. The subject sat alone at a table in front of a semicircular screen, which was enclosed by a black nonreflecting floor and ceiling. During experimental sessions, the subject held his/her head in a chinrest, to ensure a constant distance of about $70 \mathrm{~cm}$ between the eyes and the screen. Stimuli were presented on the screen through back projection by means of a Kodak Carousel projector. Timing of events and recording of choice reaction time and eye movements were controlled by an Olivetti M280 personal computer. Horizontal eye movements were measured through standard EOG techniques using silver-chloride electrodes. A warning tone was generated by an audio oscillator and was presented over headphones.
\end{abstract}

\section{Task and Stimuli}

In each trial, two stimuli were successively presented: the first was presented $22.5^{\circ}$ to the left (SL) and the second was presented $22.5^{\circ}$ to the right (SR) of the subject's meridian. A trial started with an auditory warning signal $(750 \mathrm{~Hz}, 500 \mathrm{msec}, 70 \mathrm{~dB})$, which was accompanied by a red fixation light located $3.5 \mathrm{~cm}$ below SL's position that remained on for $1.5 \mathrm{sec}$. The offset of the fixation light was immediately followed by SL and, with a stimulus onset asynchronity (SOA) of $250 \mathrm{msec}$, by SR. Both signals remained visible until the response was completed, or until a presentation duration of $2,000 \mathrm{msec}$ for $\mathrm{SL}$ and $1,750 \mathrm{msec}$ for SR was exceeded. At the start of a trial, the subject fixated the left position. After viewing SL, the eyes were shifted to SR, whereupon the subject decided on the correct response.

SL consisted of the digit 2 or 3, either intact or degraded, in all experimental conditions. A digit consisted of a dot pattern surrounded by a rectangular frame, which also consisted of dots. Digits were degraded by placing 10 dots from the frame at random positions within the frame, at positions that were not occupied by dots from the digit. There were three degraded versions of each digit.

SR differed among experimental conditions. In one condition, SR was also the digit 2 or 3 . The task was to compare SL and SR and to decide whether they were same or different. For same pairs, a left-side response button had to be pressed; for different pairs, a right-side response button had to be pressed. The second condition consisted of a summation task; SR was either a 4 or a 5 . The subjects were asked to add SL and SR; they were instructed to press the left-side button when the sum of 
SL and SR was six or eight and to press the right-side button when the sum was seven. In the third condition, the subjects decided whether the smallest digit (the 2) had been presented. SR indicated the response mapping of SL for the current trial by showing two adjacent dots representing the response buttons. One of the dots had the word yes underneath it, indicating that this button had to be pressed when SL consisted of a 2 . The other dot had the word no written above it, indicating the button to be pressed if SL had been a 3. Each digit was randomly, but equally often, assigned to the left and the right button. In the final condition, SR was an uppercase $G$ or $L$, which indicated whether or not a response to $S L$ had to be carried out ( $G=$ go, $L=$ no go). A response had to be carried out in $50 \%$ of the trials. In this condition, the left button was pressed when SL was a 2 and the right button was pressed when SL was a 3 . Throughout the experiment, the probability of each response was equal.

\section{Design and Procedure}

The study consisted of a within-subject three-factor factorial design, with condition (same-different vs. summation vs. response mapping vs. go-no go), signal quality (intact vs. degraded), and replications (the first vs. the second time a condition was performed) as main factors. All variables were varied between blocks of trials. The order of conditions was arranged in a Latin square, with the constraint that the intact and degraded version of each condition be carried out in successive blocks.

The subjects were instructed to respond optimally fast and correct. Prior to the experimental session, they first practiced naming the degraded stimuli. This was followed by 15 practice trials in each condition, during which frequent feedback of results was given. Then, the subjects performed the main experiment. An experimental block always consisted of $\mathbf{3 0}$ trials, except for the go-no go condition in which $\mathbf{6 0}$ trials were run because a response was executed on only half of the trials. Each subject completed the experiment in a session of about $4 \mathrm{~h}$.

\section{Subjects}

The subjects were 8 students ( 7 females, 1 male), aged 20-25. All reported having normal or corrected-to-normal vision. They received a small payment for participation.

\section{RESULTS}

The experimental results are summarized in Table 1 , which shows for each condition the mean reaction time to SL (TL) for intact and degraded signals, the mean duration of the saccade to SR (TM), and the fixation time of SR until the response was executed (TR).

Error rates for the individual conditions proved to be all in the range of $3.4 \%$ to $6.0 \%$. Since the percentages were low and their range was small, they were not analyzed further. A separate analysis of variance was carried out for each of the dependent variables (TL, TM, and TR). There was a main effect of signal quality on TL $[F(1,7)=71.47, p<.001]$, degraded signals being pro-

Table 1

Mean TL, TM, and TR for Each of the Experimental Tasks

\begin{tabular}{lcccc}
\hline & \multicolumn{4}{c}{ Task } \\
\cline { 2 - 5 } & $\begin{array}{c}\text { Same- } \\
\text { Different }\end{array}$ & Summation & $\begin{array}{c}\text { Response } \\
\text { Mapping }\end{array}$ & Go-No Go \\
\hline TL (Intact) & 220 & 233 & 234 & 230 \\
TL (Degraded) & 288 & 307 & 306 & 302 \\
TM & 133 & 134 & 137 & 132 \\
TR & 465 & 538 & 569 & 364 \\
\hline
\end{tabular}

Note-TL = mean fixation time to the left signal; $T M=$ mean duration of the saccade to the right signal; TR = mean fixation time of the right signal until the response was executed. cessed more slowly than intact ones ( $301 \mathrm{vs} .232 \mathrm{msec}$ ). Condition had no effect on TL $[F(3,21)=1.77, p>.05]$. TM had neither significant main effects nor interactions. With respect to TR, there was a significant main effect of condition $[F(3,21)=71.91, p<.001]$ and a significant second-order interaction of condition $\times$ signal quality $\times$ replication $[F(3,21)=7.60, p<.05]$. According to Bonferroni ( $\alpha=.05$ ), $t$ tests were carried out to compare the mean TR of the conditions in pairs, in order to further specify the main effect. It appeared that all means differed significantly from each other, except for the summation and the response-mapping conditions $[t(62)=$ $1.42, p>.008$ ]. Since differences among the TRs of qualitatively different conditions cannot be meaningfully interpreted, the second-order interaction will not be further considered.

\section{DISCUSSION}

The present experiment aimed at contributing to two related questions. The first was whether completing stimulus encoding during fixation of SL occurs irrespective of the type of task at SR. The answer seems positive, since in all conditions the effect of signal encoding was fully reflected in TL. Thus, previous findings by Sanders and Houtmans (1985) and Hansen and Sanders (1988) are not a result of their use of a same-different task but can be generalized to different experimental situations. There are no indications that stimulus encoding was completed during fixation of SL in order to avoid processing overload during TR, since processing load at SR differed considerably in various conditions of the present experiment (from $364 \mathrm{msec}$ in the go-no-go task to $569 \mathrm{msec}$ in the response-mapping task; see Table 1), but it did not affect TL. The second question was whether postperceptual processes are also tied to stimulus fixation. This seems not to be the case: TL appeared unaffected by additional demands of stimulus classification and response selection. This result is consistent with findings by Boer and van der Weijgert (1988), who found that target set size in a target-nontarget classification task has only a small effect on TL. It is also consistent with a study by van Duren (1991), who found that whether or not target classification of SL is completed during its fixation can be manipulated by instruction. Together, these results confirm that initiating the saccade is tied bottom-up to the completion of perceptual processing, whereas postperceptual processes are under top-down strategic control. From the present experiment, it cannot be concluded whether postperceptual processing occurs during the saccade or during the fixation of SR. This question will be addressed elsewhere (van Duren, 1991).

\section{REFERENCES}

Boer, L. C., \& VAN der Weijgert, E. C. M. (1988). Eye movements and stages of processing. Acta Psychologica, 67, 3-17.

EhrLich, S. F., RAYNer, K. (1981). Contextual effects on word perception and eye movements during reading. Joumal of Verbal Learning \& Verbal Behavior, 20, 641-655.

HaNSEN, W., SANDERS, A. F. (1988). On the output of encoding during stimulus fixation. Acta Psychologica, 69, 95-107.

INHOFF, W. A. (1984). Two stages of word processing during eye fixations in the reading of prose. Journal of Verbal Learning \& Verbal Behavior, 23, 457-464.

IRWIN, D. E. (1991). Information integration across saccadic eye movements. Cognitive Psychology, 23, 420-456.

IRWIN, D. E. (1992). Memory for position and identity across eye movements. Journal of Experimental Psychology: Learning, Memory, \& Cognition, 18, 307-317.

MilleR, J. O. (1988). Discrete and continuous models of human information processing: Theoretical distinctions and emperical results. Acta Psychologica, 67, 191-257. 
Posner, M. I. (1978). Chronometric explorations of mind. Hillsdale, NJ: Erlbaum.

Rayner, K., \& Pollatsex, A. (1987). Eye movements in reading a tutorial review. In M. Coltheart (Ed.), Attention and performance XII: The psychology of reading (pp. 327-362). Hillsdale, NJ: Erlbaum. Russo, J. E. (1978). Adaptation of cognitive processes to the eye movement system. In J. W. Senders, D. F. Fischer, \& R. A. Monty (Eds.), Eye movements and the higher psychological functions (pp. 89-112). Hillsdale, NJ: Erlbaum.

SANDERS, A. F. (1990). Issues and trends in the debate on discrete vs. continuous processing of information. Acta Psychologica, 74, 123-167.
Sanders, A. F., \& Houtmans, M. J. M. (1985). There is no central stimulus encoding during saccadic eye shifts: A case against general parallel processing models. Acta Psychologica, 60, 323-338.

VAN DUREN, L. L. (1991, September). Central stimulus processing during saccadic eye movements. Paper presented at the 6th European Conference on Eye Movements, Leuven, Belgium.

ZolA, D. (1984). Redundancy and word perception during reading. Perception \& Psychophysics, 36, 277-284.

(Manuscript received February 22, 1992.)

\section{Values and Ethics Series}

\section{Loyola University of Chicago and Loyola University Press}

Loyola University of Chicago and Loyola University Press have scheduled publication of Volumes IV through VII in their joint Values and Ethics Series. For 1992-93, the publication schedule includes works on the narrative theology of St. Theresa of Avila, abortion based on prenatal diagnosis, antinomies of social theory, and the burdens of distributive justice.

Interested authors in humanities, history, philosophy, theology, law, education, political science, sociology, medicine, and the helping professions, etc., should send letters of inquiry, CV, an outline of the manuscript, Table of Contents, etc., to

Dr. Rosanne Perez-Woods, Chairperson

Editorial Board, Values and Ethics Series

Loyola University of Chicago Medical Center

2160 South First Avenue, .Building 131-N

Maywood, Illinois 60153 